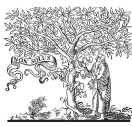

ELSEVIER

Ricevuto il:

25 febbraio 2011

Accettato il:

20 maggio 2011

Disponibile online: $x x$ xx xxxx

\section{Approccio terapeutico al paziente in terapia con bisfosfonati orali e affetto da cisti naso-palatina:}

\section{caso clinico}

\section{The therapeutic approach to patients with nasopalatine duct cysts who are receiving oral bisphosphonates: a case report}

F. Carini, , G. Porcaro ${ }^{\text {b }}$, D. Monai ${ }^{\text {b }}$, L. Barbano ${ }^{\text {c," }}$, C. Bucaloc, F. Pirrone

Hinta Responsabile Chirurgia Orale, Ospedale San Gerardo Monza (MB); Ricercatore Confermato, Scuola

HAr di Specializzazione in Chirurgia Odontostomatologica, Università degli Studi Milano-Bicocca Monza (MB)

b Specialista in Chirurgia Odontostomatologica, Università degli Studi Milano-Bicocca Monza (MB)

"Specializzando presso la Scuola di Specializzazione in Chirurgia Odontostomatologica,

w1- Università degli Studi Milano-Bicocca Monza (MB)

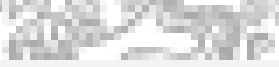
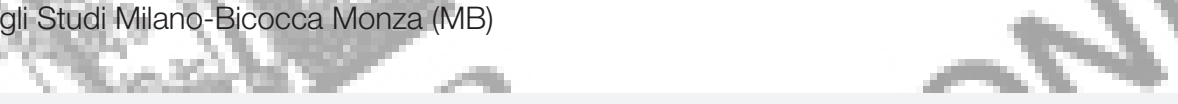

Parole chiave:

Cisti naso-palatina

Bisfosfonati orali

Alendronato

Osteonecrosi da

bisfosfonati

Chirurgia orale

Key words:

Nasopalatine duct cyst

Oral bisphosphonates

Alendronate

Bisphosphonate-related

osteonecrosis

Oral surgery
*Autore di riferimento:

barbano.lorena@

gmail.com

(L. Barbano)

\section{Riassunto}

Obiettivi: Nel presente studio viene descritto un caso di enucleazione di cisti naso-palatina in una paziente in terapia con bisfosfonati orali, farmaci sono in grado di influenzare il piano di trattamento odontoiatrico alterando la fisiologica guarigione dei tessuti.

Materiali e metodi: Attraverso un approccio chirurgico, è stata enucleata la lesione cistica nasopalatina in una paziente in terapia con bisfosfonati orali. Si rende necessaria una visione olistica del paziente in esame e una sinergica collaborazione con gli specialisti presso cui i pazienti in questione sono in cura.

Risultati: La paziente è stata rivalutata 10 giorni dopo l'intervento chirurgico e la guarigione delle mucose è risultata nella norma. La donna è stata quindi inserita in un attento programma di follow-up.

Conclusioni: Dato il rischio di mancata guarigione dei tessuti duri e molli orali in pazienti in terapia con bisfosfonati sottoposti a manovre invasive di chirurgia orale, è fondamentale somministrare una corretta profilassi antibiotica nella settimana precedente e seguente l'intervento chirurgico.

(C) 2011 Elsevier Srl. Tutti i diritti riservati.

\section{Abstract}

Objectives: The authors describe the enucleation of a nasopalatine duct cyst in a patient receiving oral bisphosphonate therapy, which can influence the outcome of dental treatments by altering the physiological tissue healing process.

Materials and methods: A surgical approach was used to remove a nasopalatine duct cyst in a patient receiving oral bisphosphonate therapy. Management requires a holistic view of the case in collaboration with the patient's physicians. Results: Ten days after surgery, the patient was reassessed, and the mucous membrane was found to be healing normally. The patient was monitored with a specific follow-up protocol.

Conclusions: Non-healing of hard and soft tissues is a risk in patients receiving oral bisphosphonate who undergo invasive oral surgery procedures. Thus, an appropriate antibiotic prophylaxis is essential before and after surgery.

(c) 2011 Elsevier Srl. All rights reserved. 


\section{F. Carini et al.}

\section{CLINICAL IMPLICATIONS}

Secondo varie review della letteratura, l'assunzione di bisfosfonati è associata a un maggior rischio di osteonecrosi dei mascellari in presenza di patologie orali che necessitano di un trattamento chirurgico. Dunque, i pazienti in terapia con bisfosfonati possono essere considerati a rischio e pertanto richiedono una maggior attenzione in termini di gestione pre-, peri- e postoperatoria.

Systematic reviews of the literature indicate that oral bisphosphonate therapy is associated with an increased risk of osteonecrosis of the jaw in patients with oral diseases requiring surgical treatment. Thus, patients taking bisphosphonates can be considered at risk for osteonecrosis. As such, they require more attention during the pre-, peri- and postoperative phases.

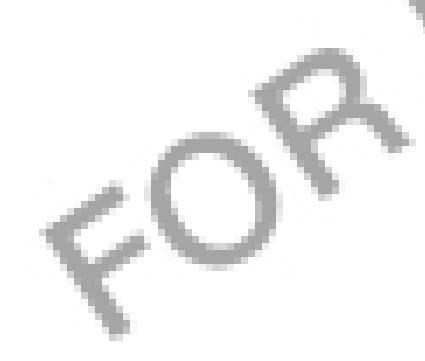

Introduzione

I bisfosfonati son solo una

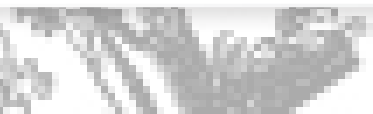
Una classe di farmaci in grado di influenzare non solo il metabolismo osseo, andando a interferire con l'attività biologica degli osteoclasti, maănche il turnover endoteliale a livello dei tessuti orali [1,2]. Data la modalità di azione dei bisfosfonati, è possibile dedurre che siano in grado di rallentare la guarigione delle ferite chirurgiche, qualora questi pazienti vengano sottoposti a interventi dichirurgia orale [1-5].

Due sono le principali modalità di somministrazione dei bisfosfonati: orale per la cura dell'osteoporosi e parenterale per la cura di mieloma multiplo, metastasi ossee, ipercalcemia maligna e morbo di Paget.

Negli ultimi otto anni, i bisfosfonati hanno ricevuto una progressiva considerazione da parte dei chirurghi orali a causa delle aumentate segnalazioni di osteonecrosi dei mascellari (osteonecrosis of the jaw, ONJ) in seguito a interventi di chirurgia orale [4]. L'ONJ da bisfosfonati, condizione descritta per la prima volta da Marx nel 2003 [6], è un'invalidante complicanza odontoiatrica che si manifesta a carico delle ossa mascellari in pazienti in terapia con bisfosfonati, da prevenire mediante precisi comportamenti preoperatori.

La ragione per cui i bisfosfonati hanno una maggior attività a carico delle ossa mascellari, non essendo queste ultime interessate principalmente da osteoporosi, risiede nel fatto che le ossa alveolari dei mascellari sono caratterizzate da una più elevata captazione di bisfosfonati, i quali in esse si concentrano rapidamente e in quantità elevate. Se il paziente ha assorbito $\mathrm{o}$ accumulato una sufficiente quantità di bisfosfonati, mediante valutazioni radiografiche è possibile osservare un mancato rimodellamento della lamina dura e una sua ipermineralizzazione, associati a un ampliamento dello spazio del legamento parodontale [7]. L'azione biologica dei bisfosfonati induce una riduzione della formazione di unità multicellulari di base, costituite da piccole isole di osteoclasti, osteoblasti e vasi ematici adiacenti [8]. - In particolare, l'azione irreversibile dei bisfosfonati a carico degli osteoclasti consiste nell'apoptosi o nella morte immediata della cellula bersaglio stessa.

Tra i bisfosfonati assunti per via orale, alendronato (che possiede un'emivita superiore a 10 anni) è uno dei farmaci più impiegati per il trattamento dell'osteoporosi e dell'osteopenia ed è efficace su tutte le fratture in prevenzione primaria e secondaria in donne a rischio molto elevato.

Le prime prove di efficacia di un bisfosfonato su grandi numeri nella prevenzione delle fratture riguardano alendronato. I risultati sono stati raggiunti inizialmente in donne con una storia di fratture vertebrali $[9,10]$ e successivamente studi di adeguata potenza ne hanno dimostrato l'efficacia sulle fratture da fragilità in generale e in prevenzione primaria (cioè donne che non avevano subito fratture in precedenza) [11]. L'effetto è evidente soprattutto sulle fratture vertebrali, ma nelle donne con demineralizzazione più accentuata ( $T$ score medio del campione in esame -3,13) anche il rischio di fratture non vertebrali risulta ridotto in modo significativo da un trattamento protratto per quattro anni in prevenzione primaria.

Una recente revisione Cochrane [12] ha valutato tutti gli studi clinici, giungendo alla conclusione che la somministrazione orale giornaliera di $10 \mathrm{mg}$ è efficace sia 
nella prevenzione primaria sia in quella secondaria. La monodose settimanale di $70 \mathrm{mg}$ possiede al momento solo documentazioni di equivalenza rispetto alla dose giornaliera in riferimento a esiti surrogati: $\mathrm{BMD}$, marcatori di riassorbimento-neoformazione ossea [13]. Uno studio condotto su una popolazione mista di 241 uomini con osteoporosi, composta per metà da pazienti già fratturati e da soggetti a rischio in prevenzione primaria, ha rilevato una riduzione del rischio di fratture vertebrali nel gruppo trattato per due anni con alendronato 10 mg/die; nel maschio non sono disponibili dati in prevenzione primaria o sull' effetto preventivo in sedinon vertebrali [14]. preventivo in sedi non vertebrali [14].
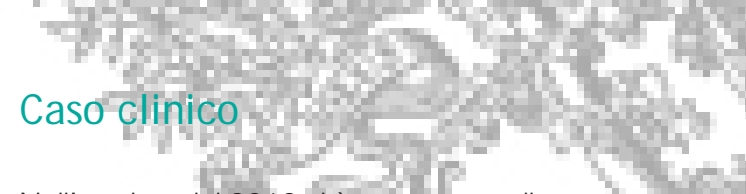

Nell'ottobre del 2010 si è presentata alla nostra attenzione, presso la Clinica Odontoiatrica dell'Ospedale San Gerardo di Monza, una paziente di 70 anni, lamentando fastidio a livello della porzione mediana del mascellare superiore.

All'anamnesi la paziente ha dichiarato l'assunzione settimanale di alendronato $70 \mathrm{mg}$ da 4 anni, nonché l'assunzione quotidiana di levotiroxina sodica $100 \mu \mathrm{g}$, propafenone cloridrato $300 \mathrm{mg}$ e furosemide sodico $500 \mathrm{mg}$. La donna presentava allergie alle penicilline. All'esame clinico è stata rilevata la presenza di una mucosa vestibolare e palatale sana e integra, mentre alla palpazione (in particolare del versante palatale) è stata notata una leggera tumefazione dalla consistenza fluttuante data dalla presenza di una lesione, confermata dall'esame radiografico, che nell'espansione nella regione naso-palatina aveva eroso la corticale palatale (fig. 1).

All'esame radiografico è stata confermata la presenza di una lesione naso-palatina radiotrasparente, tondeggiante, a margini netti, caratterizzata da un tipico orletto sclerotico più radiopaco delimitante la lesione stessa, localizzata a livello interincisivo. L'analisi radiografica è stata eseguita mediante lo studio dapprima di un'ortopantomografia delle arcate dentarie (fig. 2), che non è risultata sufficiente per la valutazione della lesione producendo un'immagine bidimensionale, e di una TC Dentascan dell'arcata dentaria superiore, che mediante la sezione assiale (figg. 3 e 4) ha messo in evidenza le conseguenze dell'espansione della lesione, ovvero
Fig. 1

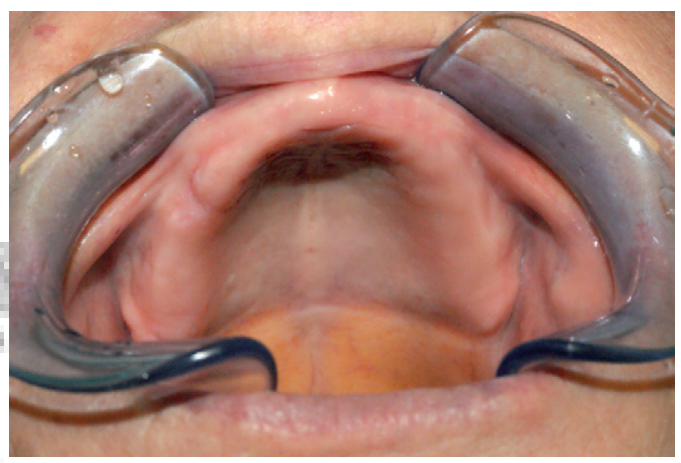

Fig. 1

Situazione clinica preoperatoria.

Fig. 2

OPT preoperatoria.

Fig. 3

TC Dentascan

sezione assiale.
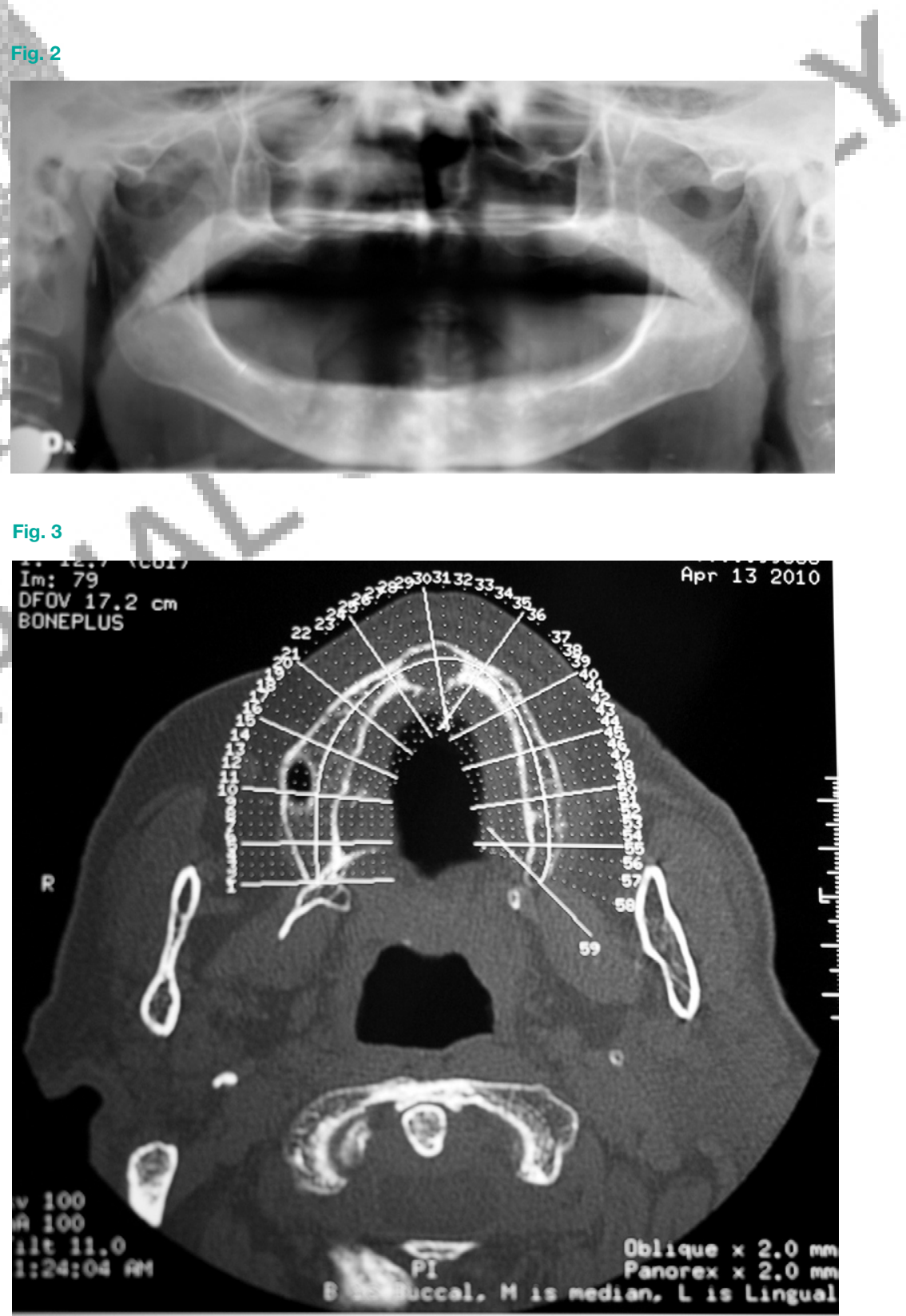


\section{F. Carini et al.}

Fig. 4

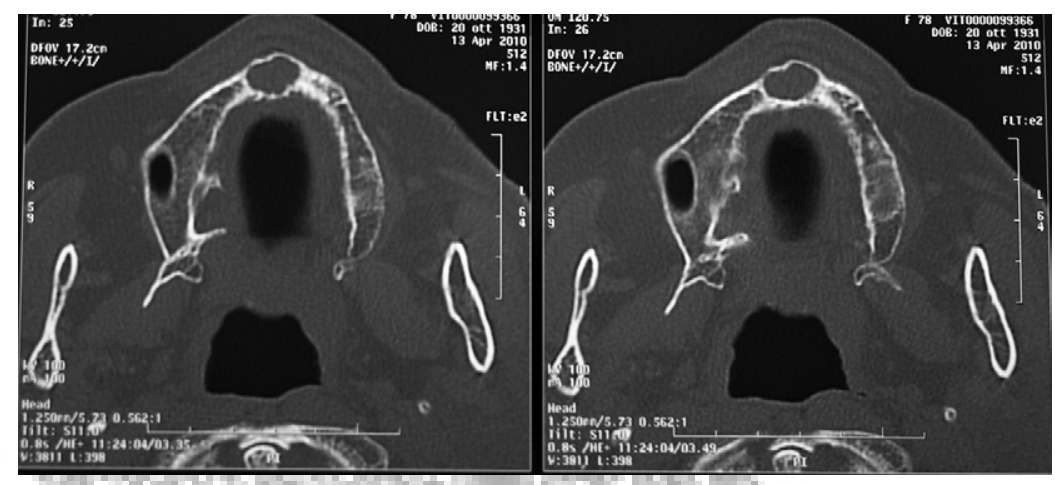

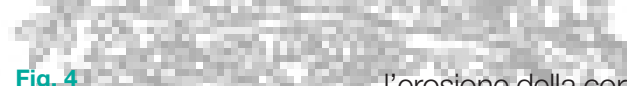

Fig. 4 T'erosione della corticale ossea palatale e il manteniTC Dentascan $\quad$ mento dell'integrità della corticale ossea vestibolare. sezione assiale. $\quad$ Le sezioni cross (figg. 5 e 6) e la sezione panorex 17 (fig. 7) hanno invece reso ben visibile l'estensione 4 della lesione e i rapporti che essa contraeva con il canale naso-palatino e le strutture anatomiche in esso contenute.

Eseguiti attentamente una corretta anamnesi e un esame clinico e radiografico è stata posta diagnosi

Fig. 5

\section{TC Dentascan} sezioni cross.

Fig. 5

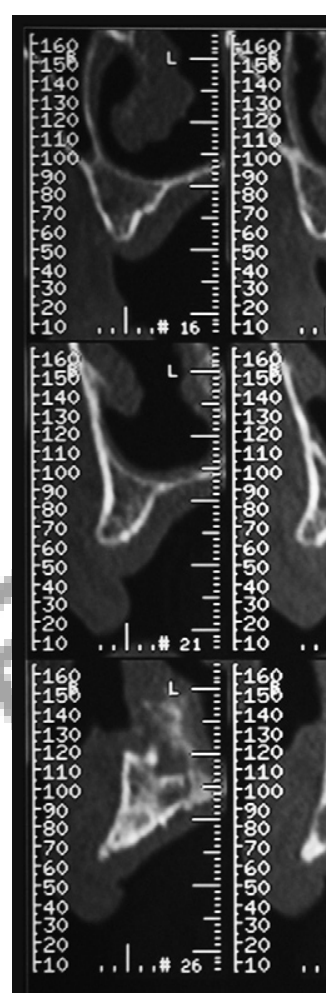
presunta di cisti naso-palatina, grazie anche alla sede anatomica tipica e all'epidemiologia della sede
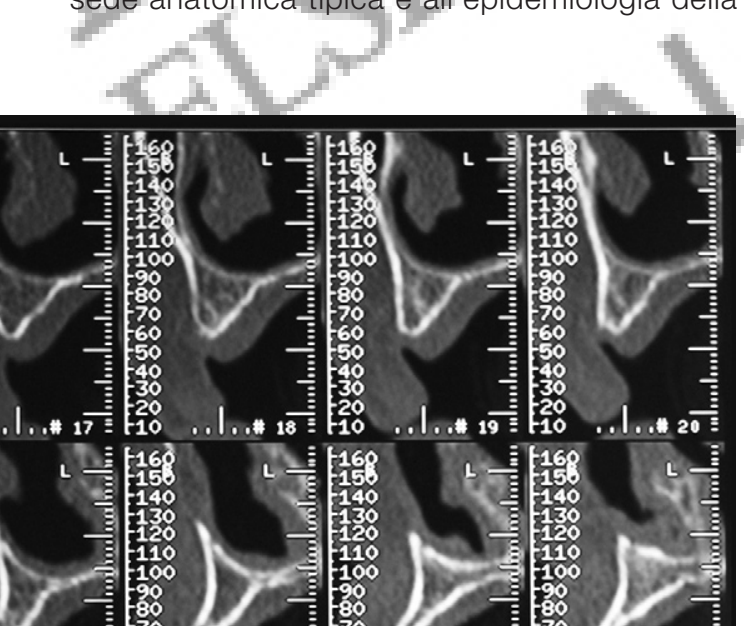

itic
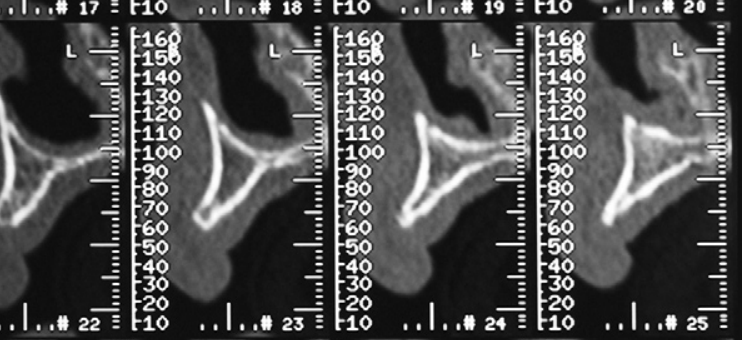

.
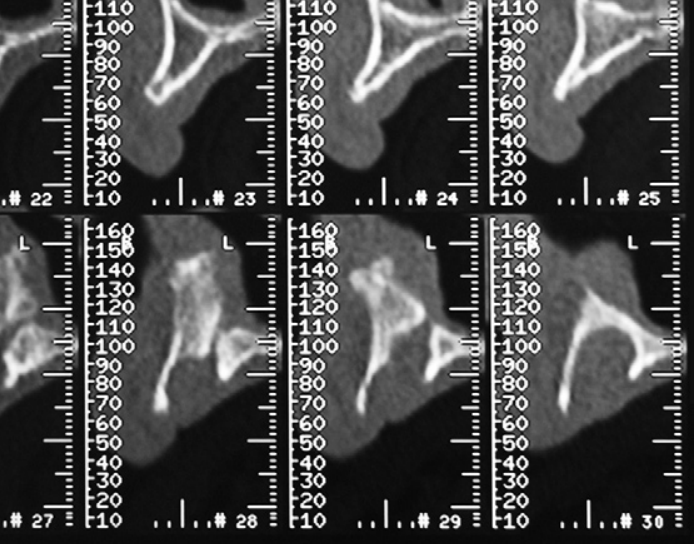

lesione. La diagnosi era presunta perché solo l'esame istologico del pezzo operatorio dopo la rimozione consente di porre una diagnosi precisa.

La cisti naso-palatina è la più comune tra le cisti fissurali, identificata frequentemente tra la quarta e la sesta decade di vita.

È stata dunque programmata l'enucleazione della lesione cistica in regime ambulatoriale di day hospital previa somministrazione, nella settimana precedente l'intervento chirurgico, di una profilassi antibiotica sistemica consistente in claritromicina in compresse da $500 \mathrm{mg}$ ogni 8 ore e metronidazolo in compresse da $250 \mathrm{mg}$ ogni 8 ore, omeprazolo in compresse da $20 \mathrm{mg}$ una volta al giorno per tutta la durata della terapia antibiotica, associati ad applicazioni di clorexidina gel $0,5 \%$. È stata inoltre continuata la stessa profilassi anche nella settimana seguente l'intervento chirurgico [15]. Questa profilassi antibiotica e antisettica è stata adottata per ridurre il rischio di ONJ da bisfosfonati [16], data la possibile guarigione ritardata delle ferite chirurgiche in pazienti definiti a rischio nel Position Paper dell'American Association of Oral and Maxillofacial Surgeons [17], poiché i bisfosfonati agiscono non solo a carico degli osteoclasti [8], ma anche a livello delle cellule endoteliali $[1,2]$.

Occorre specificare che una corretta profilassi antibiotica è una condizione necessaria, ma non sufficiente per ridurre il rischio di ONJ: nei pazienti in terapia con bisfosfonati orali, infatti, l'intervento chirurgico stesso deve essere basato su criteri di minima invasività chirurgica e di massima eradicazione, così da rimuovere tutti i potenziali focolai infettivi. È inoltre necessario ottenere una corretta giustapposizione dei lembi chirurgici con la sutura e quindi una guarigione per prima intenzione.

Previa anestesia tronculare nel nervo naso-palatino associata a un'anestesia plessica vestibolare di mepivacaina con adrenalina 1:100.000 mediante l'ausilio di una sonda parodontale PCPUNC 15, è stato possibile misurare l'estensione della lesione cistica: l'estensione verticale della cisti a partire dalla gengiva marginale era $15 \mathrm{~mm}$ circa (fig. 8), la sua estensione orizzontale $10 \mathrm{~mm}$ circa (fig. 9). Definiti i limiti della lesione, si è proceduto al disegno di un lembo di accesso mucoperiosteo a spessore totale da 1.2 a 2.3 , che ha reso possibile la visualizzazione 
a cielo aperto della lesione cistica e l'erosione della corticale palatale (fig. 10). Successivamente, con l'impiego di scollatori è stato eseguito con estrema cautela, data la mancanza di un piano di clivaggio, lo scollamento del lembo partendo dalle zone più lontane dall'area di completa erosione della corticale: in tal modo, si evita di lesionare la parete cistica garantendo l'enucleazione della lesione in monoblocco e dunque un più semplice e veloce atto chirurgico. La rimozione in monoblocco può essere utile per ridurre il rischio di escissione incompleta, ma ciò non è sempre eseguibile (ad es., in presenza di cisti con pareti sottili e molto adese alle pareti ossee). Anche se rimossa in frammenti, il trattamento può essere efficace purché si esegua un'attenta e corretta revisione della cavità residua. Individuata la parete cistica, si procede al clivaggio della lesione dalle pareti ossee. Mediante l'uso di un porta-aghi si mantiene in tensione verso l'esterno la lesione cistica e, contemporaneamente, mediante scollatori, si libera la cisti dalle pareti ossee della cavità ossea in cui è contenuta, fino alla completa rimozione (fig. 11). Nel caso in questione, anche il contenuto del canale naso-palatino è stato rimosso, data l'invasione da parte della lesione del canale stesso (come è possibile osservare nelle sezioni della TC Dentascan).

Successivamente, sono stati eseguiti la revisione della cavità residua e abbondanti lavaggi con soluzione fisiologica sterile per una migliore visualizzazione del campo operatorio. Infine, sempre con l'ausilio della sonda parodontale, sono state controllate le dimensioni della cavità residua (fig. 12), che coincidevano con le dimensioni della lesione cistica misurate nel preoperatorio. L'intervento chirurgico è stato concluso con il posizionamento di una sutura 3/0 a punti continui in seta (fig. 13), mantenuta per 10 giorni circa per facilitare la giustapposizione dei lembi e dunque garantire una guarigione per prima intenzione.

La paziente è stata dimessa dopo aver controllato il mancato sanguinamento postoperatorio della ferita chirurgica. È stata raccomandata una dieta morbida e fredda per i giorni seguenti l'intervento, associata all'impiego di ghiaccio extraorale, alla profilassi antibiotica e antisettica per i seguenti sette giorni dall'intervento chirurgico ed eventuale terapia analgesica al bisogno.
Fig. 6

TC Dentascan sezioni cross.

Fig. 6

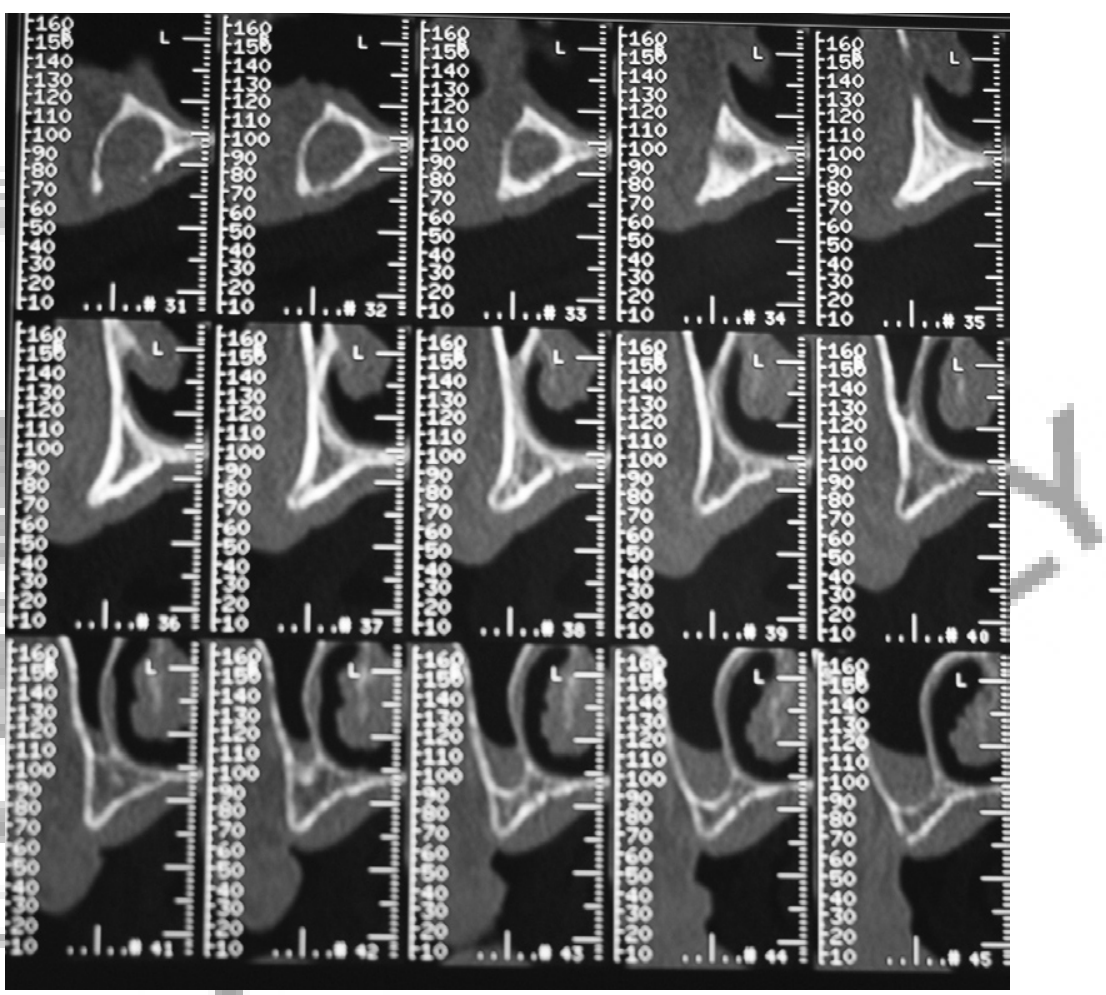

Fig. 7

TC Dentascan sezione panorex.

tris.

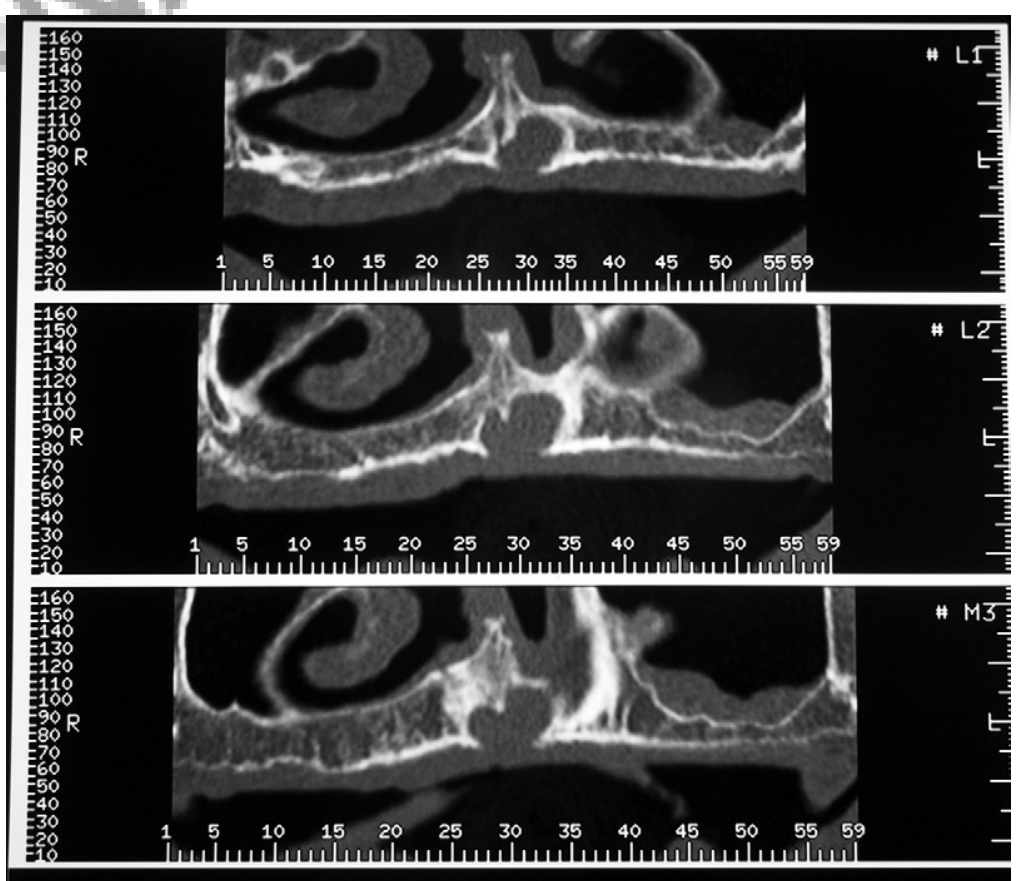




\section{F. Carini et al.}

Fig. 8

Visualizzazione clinica dell'estensione verticale della cisti naso-palatina.

Fig. 9

Visualizzazione clinica dell'estensione orizzontale della cisti naso-palatina.

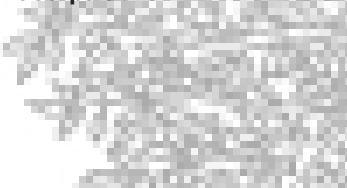
Fig. 10 mint par Lembo chirurgico di accesso.

Fig. 12

Revisione della cavità residua.

Fig. 11

Enucleazione chirurgica della cisti naso-palatina.

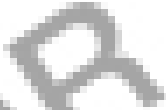

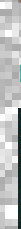

Fig. 8

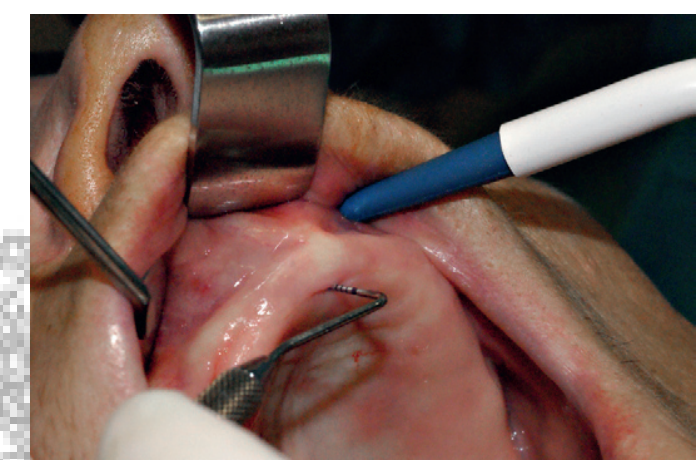

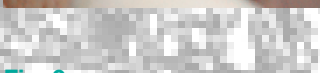

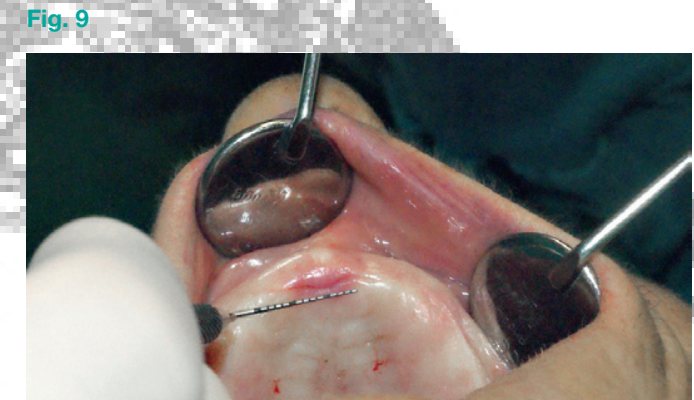

Sutura $3 / 0$.

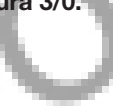

Fig. 14

Guarigione clinica a distanza di 10 giorni dall'intervento.
Fig. 12

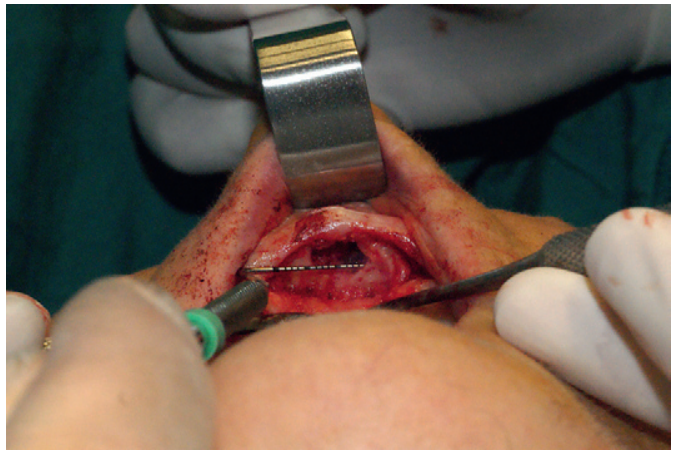

Fig. 13

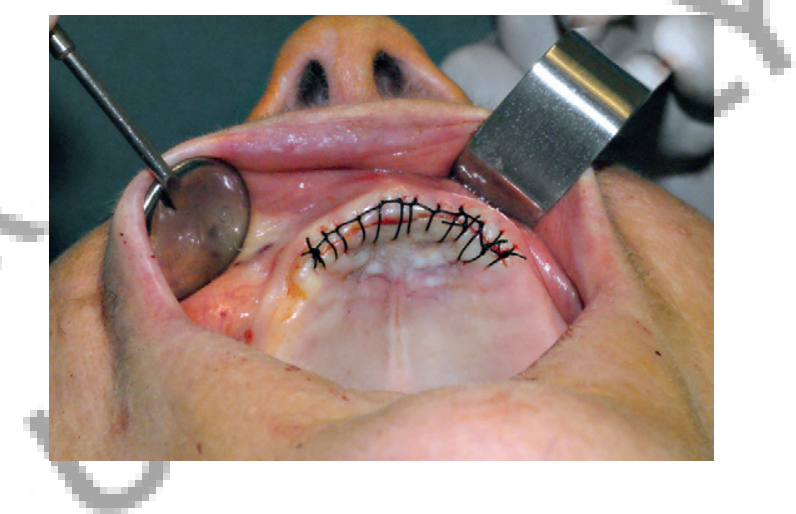

Fig. 14

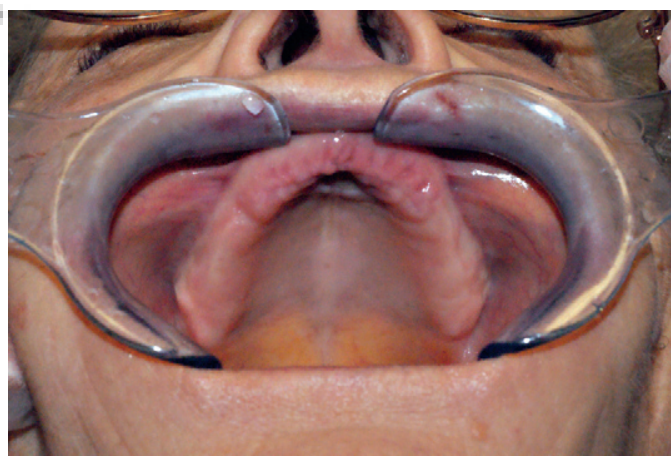

La paziente è stato rivalutata a distanza di 10 giorni dall'intervento chirurgico e si è proceduto alla rimozione dei punti di sutura. La guarigione delle mucose è risultata nella norma (fig. 14).

\section{Follow-up}

Dato il rischio di sviluppo di ONJ da bisfosfonati a distanza, la paziente è stata inserita in un programma di follow-up a 1, 3, 6 e 12 mesi, in modo da intercettare precocemente eventuali segni di ONJ

\section{6 | ITALIAN ORAL SURGERY 4/2011}

Per citare quest'articolo : Carini F, et al. Approccio terapeutico al paziente in terapia con bisfosfonati orali e affetto da cisti naso-palatina: caso clinico. Ital Oral Surg (2011), doi:10.1016/j.ios.2011.05.006 


\section{Approccio terapeutico al paziente affetto da cisti naso-palatina}

Fig. 15

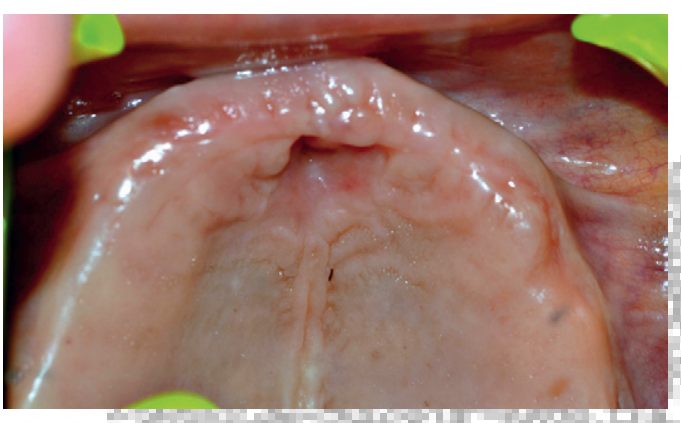

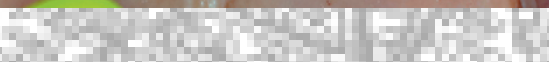
e intervenire tempestivamente. A un mese dall'intervento la paziente si è recata presso il Reparto di Odontoiatria dell'Ospedale San Gerardo per la visita di controllo (fig. 15).

Gli autori dichiarano che lo studio presentato è stato realizzato in accordo con gli standard etici stabiliti nella Dichiarazione di Helsinki e che il consenso informato è stato ottenuto da tutti i partecipanti prima del loro arruolamento allo studio.

\section{Discussione}

Dal presente studio clinico è possibile comprendere come un'attenta gestione pre-, peri-e postoperatoria sia fondamentale in pazienti in terapia con bisfosfonati orali per ottenere ottimi risultati in termini di guarigione postchirurgica.

I bisfosfonati presentano un'emivita prolungata e l'eliminazione della porzione del radicale della molecola, ovvero la catena laterale corta $\mathrm{R} 1$ che partecipa al legame con la matrice ossea mineralizzata, trattenuta nell'osso si produce nel lungo termine (in particolare, l'emivita di alendronato è di 12 anni circa) [4].

Secondo Marx et al. [18], alendronato è il più potente bisfosfonato assunto per via orale in termini di potenza e tempo di dimezzamento al pari di pamidronato disodico, dunque, tra i bisfosfonati orali, quello associato a un maggior rischio di sviluppo di ONJ. Da ciò è possibile comprendere come all'interno dei bisfosfonati per via orale sia necessario prestare attenzione anche al tipo di farmaco assunto e programmare follow-up attenti e precisi così da monitorare il paziente e riconoscere eventuali complicanze postoperatorie che richiedono un intervento tempestivo.

\section{Conclusioni}

Sebbene il rischio di $\mathrm{ONJ}$ abbia un'incidenza compresa tra lo $0,01 \%$ e lo $0,04 \%$, nei pazienti in terapia con bisfosfonati orali, qualora venisse istaurata una corretta profilassi farmacologica e l'atto chirurgico fosse basato su criteri di minima invasività chirurgica, massima eradicazione dei potenziali focolai infettivi e sull'esecuzione di una sutura corretta in modo da permettere una guarigione per prima intenzione, è fondamentale riconoscere la presenza di fattori di rischio.

I fattori in grado di incrementare in maniera significativa il rischio di ONJ da bisfosfonati orali sono la durata della somministrazione dei farmaci e l'associazione concomitante con corticosteroidi, in particolare prednisone. L'uso concomitante di corticosteroidi aumenta la tossicità dei bisfosfonati, accrescendo il rischio di sviluppo di ONJ e incrementando infine la gravità delle esposizioni ossee [19]. Da ciò è possibile concludere che l'assunzione di bisfosfonati orali rappresenta in parte un limite all'esecuzione di procedure invasive di chirurgia orale qualora non venga prestata attenzione alla gestione pre-, peri- e postoperatoria.

Il caso in questione mostra come la profilassi antibiotica e un corretto iter chirurgico rendano possibile una guarigione nella norma in pazienti in terapia con bisfosfonati orali, considerati a rischio a seguito di interventi invasivi quali l'enucleazione di una cisti.

\section{Conflitto di interessi}

Gli autori dichiarano di non aver nessun conflitto di interessi.

\section{Finanziamento allo studio}

Gli autori dichiarano di non aver ricevuto finanziamenti istituzionali per il presente studio.

\section{Bibliografia}

1. Gonzalez-Moles MA, Bagan-Sebastian JV. Alendronaterelated oral mucosa ulcerations. J Oral Pathol Med 2000;29:514-8.
Fig. 15

Guarigione clinica a distanza di 1 mese dall'intervento. 
F. Carini et al.

2. Demerjian N, Bolla G, Spreux A. Severe oral ulcerations induced by alendronate. Clin Rheumatol 1999;18:349-50.

3. Sussman G. The impact of medicines on wound healing. Pharmacist Nov 2007;26(11):874-8.

4. Sook-Bin W, Hellstein WJ, Kalmar RJ. Systematic review: bisphosphonates and osteonecrosis of the jaws. Ann Intern Med 2006;144:753-61.

5.

5. Magremanne M, Vervaet C, Dufrasne L, Duclerq I, Legrand W, Daelmans PH. Bisphosphonates et ostéo(chimio)nécrose maxillo-mandibulaire. Rev Stomatol

Hir Maxillofac 2006; 107:423-8.

4. 6. Marx RE. Pamidronate (Aredia) and zoledronate (Zometa) HHanduced avascular necrosis of the jaws: a growing epidemic.

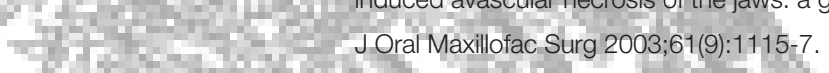
17 7. Whyte MP, Wenkert D, Clements KL, McAlister WH, 1-Rumm S. Bisphosphonate-induced osteopetrosis. N Engl J Med 2003;349:457-63.

Med 2003,349.457-63.

8. Corrado A, Cantatore FP. The bisphosphonates: chemical charactetistics, skeletal biological effects, and extra-skeletal effects. Reumatismo 2005;57(3):142-53. 9. Black DM, Cummings SR, Karpf DB, Cauley JA, Thompson DE, Nevitt MC, et al. Randomised trial of effect of alendronate on risk of fracture in women with existing vertebral fractures. Fracture Intervention Trial Research Group. Lancet 1996;348:1535-41.

10. Ensrud KE, BlackDM, Palermo L, Bauer DC, Barrett-Connor E, Quandt SA, et al. Treatment with alendronate prevents fractures in women at highest risk. Arch Intern Med 1997;157:2617-24.

11. Cummings SR, Black DM, Thompson DE, Applegate WB, Barrett-Connor E, Musliner TA, et al. Effect of alendronate on risk of fracture in women with low bone density but without vertebral fractures: results from the Fracture Intervention Trial. JAMA 1998;280:2077-82.
12. Wells GA, Cranney A, Peterson J, Boucher M, Shea B, Robinson $\mathrm{V}$, et al. Alendronate for the primary and secondary prevention of osteoporotic fractures in postmenopausal women. Cochrane Database of Systematic Reviews(1) 2008:CD001155.

13. Schnitzer T, Bone HG, Crepaldi G, Adami S, McClung M, et al. Therapeutic equivalence of alendronate $70 \mathrm{mg}$ once weekly and alendronate $10 \mathrm{mg}$ daily in the treatment of osteoporosis. Alendronate Once-Weekly Study Group. Aging (Milano) 2000;12:1-12.

14. Orwoll E, Ettinger M, Weiss S, Miller P, Kendler D, Graham J, et al. Alendronate for the treatment of osteoporosis in men. N Engl J Med 2000;343:604-10. 15. Sannino C. La chirurgia orale nel paziente con patologie sistemiche: linee guida diagnostiche e procedurali. Atti del Corso di Aggiornamento SICOI - 15 marzo 2008, Camaiore. http://www.sicoi.it/attachments/069_1619d_ Abstract_Sannino.pdf.

16. Marx RE, Sawatari Y, Fortin M, Broumand V.

Bisphosphonate-induced exposed bone (osteonecrosis/ osteopetrosis) of the jaws: risk factors, recognition, prevention, and treatment. J Oral Maxillofac Surg 2005;63:1567-75.

17. American Association of Oral, Maxillofacial Surgeons (AAOMS). Position Paper on bisphosphonate-related osteonecrosis of the jaws. J Oral Maxillofac Surg 2007;65:369-76.

18. Marx RE, Cillo JE, Ulloa JJ. Oral bisphosphonateinduced osteonecrosis: risk factors, prediction of risk using serum CTX testing, prevention, and treatment. J Oral Maxillofac Surg 2007;65(12):2397-410.

19. Bamias A, Kastritis E, Bamia C, Lia A.

Osteonecrosis of the jaw in cancer after treatment with bisphosphonates: incidence and risk factors. J Clin Oncol 2005;23:8580-7. 